\title{
Hand Digit 3
}

National Cancer Institute

\section{Source}

National Cancer Institute. Hand Digit 3. NCI Thesaurus. Code C52836.

The middle or third finger from the radial side of the hand. 